\title{
SEARCH FOR DIFFUSE COSMIC GAMMA RAYS ABOVE 200 TEV WITH THE UTAH-MICHIGAN ARRAY
}

\author{
Utah-Michigan Collaboration \\ J. Matthews \\ Department of Physics, University of Michigan \\ Ann Arbor, Michigan 48109
}

\begin{abstract}
We have searched for $\gamma$-rays in the cosmic radiation above $200 \mathrm{TeV}$ using a twolevel array of scintillators designed to measure both the electromagnetic and muon content of extensive air showers. We find no evidence for an excess number of muonpoor showers and conclude that $\gamma$-rays comprise less than $0.4 \%$ of all cosmic rays above $200 \mathrm{TeV}$, and less than $0.05 \%$ above $1000 \mathrm{TeV}(90 \% \mathrm{CL})$. The muon content of showers from the direction of the Galactic disk is the same as that of showers from other regions of the sky. The ratio of the flux of Galactic $\gamma$-rays to that of cosmic rays is $<8.0 \times 10^{-5}(90 \% \mathrm{CL})$. This limit, based on muon measurements, represents a significant improvement over previous experiments and approaches predicted levels for diffuse $\gamma$-rays from the Galactic plane.
\end{abstract}

\section{INTRODUCTION}

Regardless of whether compact sources of UHE $\gamma$-rays exist, the interaction of the highest energy cosmic rays with the cosmic background radiation or with interstellar gas would generate diffuse fluxes of $\gamma$-rays. Diffuse $\gamma$-ray fluxes can be either isotropic or concentrated along the Galactic disk, depending upon the production mechanism.

If UHE cosmic rays are of extragalactic origin an isotropic flux of $\gamma$-rays will result from the interaction of cosmic ray protons above $10^{19} \mathrm{eV}$ with the cosmic background radiation (CBR). Secondary $e^{+}, e^{-}$, and $\gamma$-rays encounter other CBR photons over intergalactic distances and subsequently cascade ${ }^{1,2}$. An enhanced flux of $\gamma$-rays near $10^{14} \mathrm{eV}$ results when the cascades terminate near the $e^{+} e^{-}$production threshold.

A flux of $\gamma$-rays from the direction of the Galactic disk should arise from interactions of primary cosmic rays on gas and dust. The intensity of these $\gamma$-rays should reflect the distribution of interstellar material ${ }^{1}$. In the $30 \mathrm{MeV}$ to $5 \mathrm{GeV}$ regime, photons produced in this manner have been observed by COS-B and SAS-2 satellites and used to map the density of Galactic hydrogen ${ }^{3}$.

Recent calculations suggest that, above $100 \mathrm{TeV}$, the flux of Galactic $\gamma$-rays should dominate the isotropic diffuse flux. The ratio of the Galactic flux $I_{\gamma}$ to that of ordinary hadronic cosmic rays has been predicted to be $7 \times 10^{-5}$, from the direction of the Galactic center ${ }^{1}$. Previous experiments have not observed this flux, setting limits $I_{\gamma} / I_{C R}<1 \%$ from the Galactic plane. ${ }^{4}$ 
We have searched for diffuse $\boldsymbol{\gamma}$-ray emission using the muon content of extensive air showers to distinguish $\gamma$-ray-induced events. The validity of this approach is based on the assumption that UHE $\gamma$-rays have interaction properties which can be extrapolated from lower energies. There have been some hints that muon production in $\gamma$-showers is somehow enhanced ${ }^{5}$. This has become one of the outstanding questions of cosmic-ray physics, but the data is inconclusive and no convincing theoretical model exists. Observation of diffuse fluxes of $\boldsymbol{\gamma}$-rays by means of muon discrimination would confirm the standard model of muon photoproduction and provide a valuable "test beam" with which to calibrate the muon content of $\gamma$-showers.

\section{EVENT SELECTION}

The Utah-Michigan array is located at Dugway, Utah, at the site of the Fly's Eye installation (atmospheric depth $870 \mathrm{~g} / \mathrm{cm}^{2}$ ). This experiment was the first stage of the Utah-Michigan-Chicago (UMC) experiment and is more fully described elsewhere ${ }^{6,7}$. The present data are from 33 surface scintillator stations distributed within a circle of $100 \mathrm{~m}$ radius and 512 muon counters $\left(1280 \mathrm{~m}^{2}\right)$ buried $3 \mathrm{~m}$ below the ground in eight widely spaced patches. This is the largest muon detector ever employed in an air shower array.

The data here are restricted to showers whose total sizes $\mathrm{N}$ lie in the range $3 \times 10^{4}<\mathrm{N}<10^{6}$, cores within $100 \mathrm{~m}$ of the center of the array, and directions within $40^{\circ}$ of the zenith. We estimate ${ }^{6}$, based on detector simulations, that the $\gamma$-ray energy threshold $E_{t}$ is about $200 \mathrm{TeV}$ after these cuts. If the minimum size cut is increased to $N>3 \times 10^{5}$, then $E_{t}$ increases to about $1000 \mathrm{TeV}$.

We compute $N$ and the muon size $N_{\mu}$ by fitting the lateral distribution of surface and buried counters to standard functions ${ }^{7}$. The muon content of individual showers fluctuates about the mean value for showers of the same size and zenith angle. We define the relative muon size $R_{\mu}$ of individual showers as

$$
R_{\mu} \equiv \log _{10} \mathrm{~N}_{\mu}-\left\langle\log _{10} \mathrm{~N}_{\mu}\right\rangle
$$

where $\left\langle\log _{10} \mathrm{~N}_{\mu}\right\rangle$ is a function of $\mathrm{N}$ and $\theta$ and is computed separately for each data run of about $24 \mathrm{~h}$ duration.

The distribution of $R_{\mu}$ is shown in Figure 1. We select individual air showers as candidates for $\gamma$-ray-induced showers when the muon size is less than onetenth the expected (mean) value. Specifically, we accept showers with $R_{\mu}<-1.0$ as candidates. We find, by simulating the detector's response, that $80 \%$ of $\gamma$ showers with $\mathrm{N}>3 \times 10^{4}$ satisfy the above criterion, rising to $90 \%$ for $\mathrm{N}>10^{5}$.

\section{ISOTROPIC DIFFUSE EMISSION}

Figure 1 displays the distribution of relative muon sizes $R_{\mu}$ for data from 1988 April 3 until 1990 February $20\left(1.9 \times 10^{7}\right.$ events $)$. Events with $R_{\mu}<-1.0$ 
are mainly small showers $\left(\mathrm{N} \approx 3 \times 10^{4}\right)$. The fluctuation of the muon size for small hadronic cosmic-ray showers is substantial and the shape of the curve is in accord with simulations of proton induced cascades and muon counting statistics in the detector. We set the most conservative limits on the isotropic flux of $\gamma$-rays by taking all showers with $R_{\mu}<-1$ as candidates. The ratio of $\gamma$-rays to the total cosmic ray flux is then $I_{\gamma} / I_{C R}<4.3 \times 10^{-3}(90 \% \mathrm{CL})$ for $\mathrm{E}_{\gamma}>200 \mathrm{TeV}$. If we consider the largest showers $\left(\mathrm{E}_{\gamma}>1000 \mathrm{TeV}\right)$, the above limit is lowered to $I_{\gamma} / I_{C R}<4.8 \times 10^{-4}(90 \% \mathrm{CL})$.

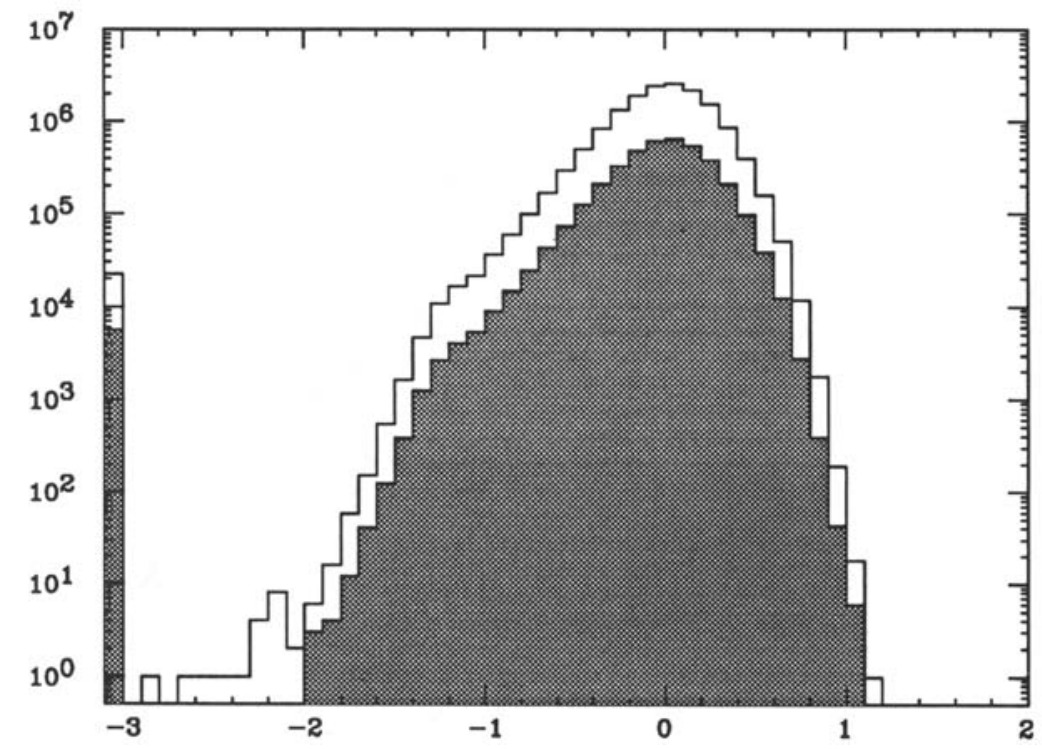

Figure 1. Distribution of relative muon size $R_{\mu}$ (Eq. 1). Data is divided into two groups: Galactic (shaded, $\left.|b|<10^{\circ}\right)$ and Non-Galactic $\left(|b|>10^{\circ}\right)$. Showers with no recorded muons are plotted as underflows in the leftmost bin.

Our sensitivity to $\gamma$-rays can be further improved by estimating the expected number of showers with $R_{\mu}<-1$ due to downward fluctuations of muon size in ordinary cosmic ray showers. We merely note here that our simulations of the detector's response to proton showers shows qualitative agreement with the shape of the $R_{\mu}$ distribution for $R_{\mu}<0$. It is not necessary to include any $\gamma$-rays in the cosmic radiation to explain the data.

\section{GALACTIC DIFFUSE EMISSION}

We search for enhanced Galactic $\gamma$-ray emission by comparing the muon content of showers from the direction of the Galactic plane with all other events 
("non-Galactic" events). We define the Galactic disk here by Galactic latitudes $\left|b^{I I}\right|<10^{\circ}$. Because of our northern latitude, this sample of events is mainly from the region $30^{\circ}<\ell^{I I}<220^{\circ}$ in Galactic longitude.

Figure 1 shows the $R_{\mu}$ distributions separately for Galactic and non-Galactic showers. Galactic data are shaded. The non-Galactic sample has about four times as many events as the Galactic data. There is no evidence for excess muon-poor showers either from the Galactic disk or non-Galactic directions. Since the majority of showers in each set are hadron induced, we predict the expected background of muon-poor showers in the Galactic data by scaling the non-Galactic distribution so that the number of showers with $R_{\mu}>0$ is the same in both samples.

We observe 19537 muon-poor showers from the Galactic disk and expect $19503 \pm 71$. With $90 \%$ confidence, there are less than 249 excess muon-poor showers from the total Galactic sample of $3.9 \times 10^{6}$ events. An upper limit on he ratio of $\gamma$-ray showers to cosmic rays is then

$$
I_{\gamma} / I_{C R}<\frac{249}{0.8 \times\left(3.9 \times 10^{6}\right)}=8.0 \times 10^{-5}
$$

for $\mathrm{E}_{\gamma}>200 \mathrm{TeV}$ and in the Galactic region defined above. Similar limits are obtained when the data set is restricted to higher energies. This limit is very close to some predicted values ${ }^{1}$.

\section{MOLECULAR CLOUD EMISSION}

Satellite observations reveal that the intensity of low energy ( $>30 \mathrm{MeV}$ ) $\gamma$-rays varies along the Galactic plane ${ }^{3}$. To the extent that these $\gamma$-rays are products of interactions of cosmic rays with gas and dust, the variation is due to nonuniformities of the interstellar medium. We find no significant excess emission of muon-poor showers $(>200 \mathrm{TeV})$ from any part of the Galactic plane visible to the detector.

Table 1 lists four northern hemisphere molecular clouds which are sources of $100 \mathrm{MeV} \gamma$-rays ${ }^{8}$. We also list regions near the Galactic center (note: the array acceptance drops off rapidly below $\ell^{I I}=30^{\circ}$ ) and the center of the Virgo cluster, where an enhanced diffuse $\gamma$ flux has been predicted ${ }^{9}$. The listed areas of the objects are the sizes of the acceptance windows used in the analysis. The energy thresholds $\mathrm{E}_{t}$ vary with the declination of the object.

No significant excess signals are seen from any of the objects listed. We compute limits on the ratio $I_{\gamma} / I_{C R}$ as described in the previous section and obtain the corresponding flux using the all-particle cosmic ray intensity $I_{C R}$ given by Protheroe ${ }^{5}$. We have included a factor which estimates the loss of signal due to reconstruction errors. The flux upper limits are generally at least ten times larger than values obtained by extrapolating satellite results to our energy thresholds ${ }^{10}$. 


\begin{tabular}{|c|c|c|c|c|c|c|}
\hline Object & $\begin{array}{c}\text { Coor } \\
\ell^{\text {II }}\end{array}$ & $\begin{array}{l}\text { inates } \\
\mathrm{b}^{\mathrm{II}} \\
\mathrm{g})\end{array}$ & $\begin{array}{c}\text { Area } \\
\left(\operatorname{deg}^{2}\right)\end{array}$ & $\begin{array}{c}\mathbf{E}_{\mathrm{t}} \\
(\mathrm{TeV})\end{array}$ & $\begin{array}{c}\mathrm{I}_{\gamma} / \mathrm{I}_{\mathrm{CR}} \\
(90 \% \mathrm{CL} \\
\text { upper limit) } \\
\left(10^{-4}\right)\end{array}$ & $\begin{array}{c}\mathrm{I}_{\gamma}>\mathrm{E}_{\mathrm{t}} \\
(90 \% \mathrm{CL} \\
\text { upper limit) } \\
\left(10^{14} \mathrm{~cm}^{-2} \mathrm{~s}^{-1}\right)\end{array}$ \\
\hline Cas OB6 & 135 & 2 & 28 & 300 & 3.8 & 0.87 \\
\hline Per OB2 & 157 & -21 & 28 & 200 & 9.6 & 4.1 \\
\hline Cygnus & 80 & 3 & 28 & 200 & 6.2 & 2.7 \\
\hline Taurus & 172 & -15 & 100 & 250 & 4.0 & 3.8 \\
\hline "Central" Gal. & $30-40$ & \pm 10 & 200 & 1100 & 9.6 & 1.5 \\
\hline Virgo (M87) & -74 & 74 & 78 & 500 & 4.7 & .95 \\
\hline
\end{tabular}

Table 1. Observations of selected molecular clouds, the central Galaxy, and Virgo.

\section{ACKNOWLEDGEMENTS}

The authors gratefully acknowledge the help and support of our colleagues at the University of Chicago and Colonel J.A. Van Prooyen and the staff of Dugway Proving Ground. This work was supported in part by the U.S. Department of Energy and the National Science Foundation.

\section{REFERENCES}

1. V.S. Berezinsky and V.A. Kudryavtsev, Astrophys. J.349, 620 (1990); V.S. Berezinsky and V.A. Kudryavtsev, Sov. Astron. Lett. 14, 370 (1988).

2. F. Halzen et al., Phys. Rev.D41, 342 (1990); J. Wdowczyk and A.W. Wolfendale, Astrophys. J.349, 35 (1990); S. Yoshida and M. Teshima,Proc. 21st Internat. Cosmic Ray Conf. (Adelaide) 2, 403 (1990).

3. H.A. Mayer-Hasselwander et al., Astron. Astrophys. 105, 164 (1982); R.C. Hartman et al.,Astrophys. J. 230, 597 (1979).

4. R.W. Clay, R.J. Protheroe, P.R. Gerhardy,Nature (London) 309, 687 (1984); K. Suga et al., Astrophys. J. 328, 1036 (1988); P.T. Reynolds et al.,Proc. 21st Internat. Cosmic Ray Conf. (Adelaide) 2, 383 (1990).

5. M. Samorski and W. Stamm,Proc. 18th Internat. Cosmic Ray Conf. (Bangalore) 11, 244 (1983); for a recent review, see R.J. Protheroe, Proc. 20th Internat. Cosmic Ray Conf. (Moscow) 8, 21 (1987).

6. D. Ciampa et al., Phys. Rev. D42, 281 (1990); D. Sinclair, Nucl. Instr. Meth. A278, 583 (1989).

7. G.L. Cassiday et al.,Proc. 21st Internat. Cosmic Ray Conf. (Adelaide) 9, 94 (1990).

8. M.R. Issa and A.W. Wolfendale, Nature (London) 292, 430 (1981).

9. J. Wdowczyk and A.W. Wolfendale,J. Phys. G 16, 1399 (1990).

10. D. Ciampa et al.,Proc. 21st Internat. Cosmic Ray Conf. (Adelaide) 2, 388 (1990). 